\title{
Perfil de usuarios con diabetes e hipertensión arterial y su relación con indicadores de resultado clínicos
}

\author{
María Elena Lagos ${ }^{a}$, Alide Salazar $^{b}$, Katia Sáez $^{c}$ \\ a Enfermera. Doctora en Enfermería. Profesor Asistente, Facultad de Enfermería, Universidad de Concepción, Chile. \\ b Enfermera-Matrona. Doctora en Enfermería. Profesor Titular, Facultad de Enfermería, Universidad de Concepción, Chile. \\ c Ingeniero Matemático. Doctor en Ingeniería Eléctrica. Profesor Asociado, Facultad de Ciencias Físicas y Matemáticas, \\ Universidad de Concepción, Chile.
}

Objetivo: Describir el perfil de usuarios con diabetes mellitus (DM) e hipertensión arterial (HTA) y su relación con indicadores de resultado clínico.

Sujetos y métodos: Estudio transversal, correlacional en usuarios con DM y/o HTA, reclutados desde 4 centros de salud de un Servicio de Salud del centro sur de Chile. Los instrumentos y mediciones incluyeron: cuestionario de variables biodemográficas, "Evaluación de la atención de enfermedades crónicas para pacientes", hemoglobina glicosilada (HbA1c), presión arterial sistólica (PAS) y diastólica (PAD).

Resultados: Se evaluaron 222 personas con HTA y/o DM con edad promedio 53,9 (DE 7,5) años, la mayoría mujeres $(67,6 \%)$. Se encontró un predominio de HTA $(53,6 \%)$, seguido de DM+HTA $(31,1 \%)$ y DM $(15.3 \%)$. El promedio de PAS y de $\mathrm{HbA} 1 \mathrm{c}$ fue levemente superior en usuarios con patología mixta (DM+HTA). El promedio de la evaluación de la atención desde la perspectiva del paciente fue 2,61 (DE 1,13), siendo mejor evaluado el "Diseño de un sistema de entrega de cuidados/ Apoyo a la decisión". Se encontró relación significativa en usuarios con HTA entre PAS y ${ }^{\circ}$ de fármacos $(\mathrm{p}=0,026)$; en personas con DM entre HbA1c y años de enfermedad $(\mathrm{p}=0,002)$ y en usuarios con multimorbilidad (HTA+ DM) entre años de enfermedad con HbA1c $(\mathrm{p}=0,01)$ y con PAS $(\mathrm{p}=0,01)$.

Conclusión: La evaluación de la atención sugiere la necesidad de mayor esfuerzo en el control de los parámetros terapéuticos, incorporando mejoras en la atención proporcionada. Se necesita más investigación para definir la relación entre el perfil de usuarios con enfermedades crónicas y los resultados clínicos.

\section{Correspondencia:}

Enf. Alide Salazar M.

Facultad de Enfermería,

Universidad de Concepción.

Fono: (56) 992185419 


\section{Diabetes and Hypertension; how user profile relates to indicators of clinical outcomes}

Aim: To decribe the profile of users of a health program for chronic diseases (CD), especifically Diabetes Mellitus (DM) and Hypertension (HT), and its relation to indicators of clinical results.

Subjects and methods: The subjects had DM and/or HT and were randomly selected form 4 health centers in southern Chile. Questionaires exploring demographic variables, Patient Assessement of Chronic Illness Care (PACIC) measurements of HbA1c, systolic (SBP) and diastolic (DBP) pressures were used as indicators of clinical results.

Results: 222 subjects with DM and/or HT were evaluated. Mean age was 53.9 years-old (SD 7.5), 67.6\% were females. The prevalence of $\mathrm{CD}$ was $53.6 \%$ for HT, $31.1 \%$ for DM+HT and $15.3 \%$ for DM. Mean BP and mean $\mathrm{HbAlc}$ were slightly higher in users with $\mathrm{DM}+\mathrm{HT}$. As perceived by the patient, the evaluation of care had a mean score 2.61 (SD 1.13). "Design of a method of care delivery / decission support" obtained the highest score. In subjects with HT there was a significant correlation between SBP and the number of drugs being received $(\mathrm{p}=0.03)$. Also, a significant correlation between $\mathrm{HbA} 1 \mathrm{c}$ and disease duration was found in subjects with DM ( $\mathrm{p}=0.02)$. In subjects with $\mathrm{DM}+\mathrm{HT}$ significant correlation $(\mathrm{p}=0.01)$ existed between disease duration and both HbA1c and SBP.

Conclusion: The evaluation of care suggests the need for a stronger effort in controlling therapeutic measures. Further studies are needed to refine the relation of user profile and clinical results

Key words: chronic disease, patient outcome assessment; type 2 diabetes; hypertension; health care, primary. 


\section{Introducción:}

Las enfermedades cardiovasculares (ECV) son una prioridad sanitaria de primer orden para la mayoría de los países del mundo ${ }^{1}$. En Chile, $84 \%$ de la carga de enfermedad obedece a las enfermedades crónicas (EC) y, de ellas, las ECV representan la principal causa de mortalidad, con un total de 25.744 defunciones en el año 2011, lo que representó el $27 \%$ del total de muertes ${ }^{2,3}$. Se destacan en este grupo las enfermedades cerebrovasculares como la primera causa específica de muerte $(34 \%)$, seguidas por las isquémicas del corazón, $(28 \%)^{4}$ y la hipertensión arterial (HTA) como la principal causa específica de carga de enfermedad, tanto en hombres como en mujeres ${ }^{3,4}$. Por su parte, la diabetes mellitus (DM) supone un grave problema de salud pública, por la alta morbimortalidad asociada, tanto por sus complicaciones microvasculares y macrovasculares, cuya prevalencia ha aumentado, estimándose para el 2035 un incremento de $12,7 \%{ }^{3,5}$.

Cabe mencionar que la cronicidad de estas condiciones no se da de forma aislada. Por el contrario, se caracteriza porque la persona puede ser afectada por más de una enfermedad crónica y/o factores de riesgo, los que coexisten e interrelacionan, dando origen a la multimorbilidad que multiplica el riesgo cardiovascular $(\mathrm{RCV})^{6}$. En consecuencia, se observa una baja proporción de personas con DM y/o HTA bien controlada, es decir, con hemoglobina glicosilada ( $\mathrm{HbA} 1 \mathrm{c})<7 \%$ y presiones arterial sistólica (PAS) y diastólica (PAD) $<40 / 90^{2,3,6}$.

En este contexto, y considerando el riesgo que esto conlleva para la salud de las personas, el Ministerio de Salud de Chile ha considerado abordar este fenómeno como una prioridad $^{2}$. Es así como el Programa de Salud Cardiovascular (PSCV) ha contribuido al aumento de cobertura para personas con $\mathrm{HTA}+\mathrm{DM}^{7}$. Así, se han formulados objetivos de Salud 2000-2010 y 2011-2020 incluyendo el objetivo estratégico $\mathrm{N}^{\circ} 2$, cuyo resultado es el logro de metas dirigidas a controlar las consecuencias de las enfermedades crónicas mencionadas. Se han propuesto Estrategias Transversales para el manejo y gestión de las EC, como la "Implementación del Modelo de Cuidados Crónicos" (MCC) ${ }^{8}$, que se fundamenta en resultados obtenidos en diversos escenarios del mundo. En ellos, se ha demostrado mejorar la calidad en salud, la seguridad, los costos y la reducción de disparidades en salud basadas en nivel socioeconómico ${ }^{9-14}$. Es así como se ha convertido en una necesidad evaluar la atención desde la perspectiva del usuario que recibe atención del equipo de salud, para lo cual el cuestionario "Evaluación de la atención de enfermedades crónicas para pacientes" recoge información sobre las acciones efectuadas por el equipo de salud y para apoyar el automanejo de las personas que padecen EC ${ }^{19-22}$

Para el diseño de futuras intervenciones es necesario disponer de una caracterización del programa de Salud Cardiovascular por parte de los usuarios incluyendo una evaluación desde su perspectiva como también de las variables asociadas a los resultados clínicos. De este modo se podrá diseñar mejor futuras intervenciones tendientes a mejorar la prevención de complicaciones en las patologías crónicas estudiadas.

El objetivo del presente estudio fue describir el perfil de usuarios con diabetes e hipertensión arterial y su relación con los indicadores de resultado clínico.

\section{Métodos:}

Diseño: Trasversal y correlacional. Unidad de análisis: Usuario con DM y/ o HTA correspondiente a un Servicio de Salud del centro sur de Chile. La selección y tamaño de la muestra se realizó mediante muestreo por conglomerados. Se consideró como conglomerados a los 11 Centros de Salud que dependen del Servicio de Salud. Se trabajó con cuatro centros de salud familiar seleccionados en forma aleatoria, dado el limitado número del personal y de los recursos comprometidos en el proyecto. El total de usuarios atendidos fue 6.014. Para la obtención de la muestra se revisó el tarjetón de control, y se seleccionaron 222 usuarios que cumplieron con los criterios de inclusión y exclusión.

Criterios de inclusión: usuarios con diabetes mellitus tipo 2 y/o hipertensión arterial; control en el programa cardiovascular por más de 6 meses; tener entre 20 y 64 años de edad, y otorgar su consentimiento informado para participar del estudio. Criterios de exclusión: usuarios con cardiopatía isquémica, accidente cerebrovascular con secuelas, EPOC moderado a severo, obesidad, enfermedad psiquiátrica, enfermedad crónica terminal, personas inmovilizadas, enfermedades tiroideas, cáncer, HIV, enfermedad reumática severa, otra patología severa que le impidiese participar y/o personas que no son capaces de leer y entender el formulario de información del paciente debido a demencia o analfabetismo.

Instrumentos/mediciones: a) Cuestionario de variables biodemográficas; b) Evaluación de la atención de enfermedades crónicas para pacientes ${ }^{19}$; c) Indicadores de resultado clínico: hemoglobina glicosilada (HbA1c), presión arterial sistólica (PAS) y presión arterial diastóli- 
ca (PAD) y d) Registro de medicamentos, obtenido desde el registro de farmacia de cada centro de salud. Los datos fueron recolectados por enfermeras/os previamente capacitados/as por un Organismo Técnico de Capacitación (OTEC). Se aplicaron cuestionarios, así como también las mediciones de HbA1c, PAS y PAD, previo consentimiento informado. La investigación fue aprobada por el Comité Ético Científico del Servicio de Salud Talcahuano (Oficio $\mathrm{N}^{\circ} 54$ ).

Procesamiento y análisis de los datos. Se realizó un análisis descriptivo e inferencial con correlación de Pearson. Se consideró como significancia estadística $\mathrm{p} \leq 0,05$.

Tabla 1.

Características biodemográficas de los participantes e indicadores clínicos $(\mathrm{N}=222)$.

\begin{tabular}{|c|c|c|}
\hline \multirow[b]{2}{*}{ Edad (años) (promedio, DE) } & \multicolumn{2}{|c|}{$\begin{array}{r}\text { Promedio (DE) } \\
n \text { (porcentaje) }\end{array}$} \\
\hline & 53,9 & 7,5 \\
\hline \multicolumn{3}{|l|}{$\operatorname{Sexo}(n, \%)$} \\
\hline Hombre & 72 & 32,4 \\
\hline Mujer & 150 & 67,6 \\
\hline \multicolumn{3}{|l|}{ Estado civil $(n, \%)$} \\
\hline Soltero(a) & 37 & 16,7 \\
\hline Casado(a) & 154 & 69,7 \\
\hline Divorciado(a) & 9 & 4,1 \\
\hline Viudo(a) & 12 & 5,4 \\
\hline Otro & 9 & 4,1 \\
\hline \multicolumn{3}{|l|}{ Escolaridad (n, \%) } \\
\hline Sin escolaridad & 1 & 0,5 \\
\hline Educación básica & 57 & 25,8 \\
\hline Educación media & 127 & 57,5 \\
\hline Instituto técnico-profesional & 21 & 9,5 \\
\hline Universitaria & 15 & 6,8 \\
\hline \multicolumn{3}{|l|}{ Patología (n, \%) } \\
\hline Solo HTA & 119 & 53,6 \\
\hline Solo DM & 34 & 15,3 \\
\hline HTA+DM & 69 & 31,1 \\
\hline \multicolumn{3}{|l|}{ Indicadores de resultado clínico } \\
\hline PAS (mmHg) & 126,91 & 15,83 \\
\hline Solo HTA & 125,9 & 15 \\
\hline $\mathrm{HTA}+\mathrm{DM}$ & 128,6 & 17,1 \\
\hline PAD (mmHg) & 80,34 & 11,4 \\
\hline Solo HTA & 80,6 & 10,6 \\
\hline HTA+DM & 79,8 & 12,8 \\
\hline Hemoglobina glicosilada (HbA1c, \%) & 7,3 & 1,9 \\
\hline Solo DM & 7,2 & 1,9 \\
\hline $\mathrm{HTA}+\mathrm{DM}$ & 7,4 & 1,9 \\
\hline
\end{tabular}

Solo HTA (n=119); Sólo DM (n=34); HTA + DM (n=69).

\section{Resultados:}

Características biodemográficas e indicadores clínicos. El promedio de edad fue 53,9 (DE 7,5) años. Los participantes fueron principalmente mujeres $(67,6 \%)$. El estado civil predominante correspondió a casados $(69,7 \%)$. Respecto de la escolaridad, destaca el porcentaje de educación media $(57,5 \%)$, seguido de educación básica $(25,8 \%)$. En cuanto a la distribución de EC, más de la mitad de las personas tenía HTA $(53,6 \%)$, solo $15,3 \%$ padecía DM y $31,1 \%$ tenía multimorbilidad (HTA + DM). El promedio de años de EC fue de 7, 06 años (DE 6,33). Entre los indicadores de resultado clínico, el promedio global de PAS fue 126,91 mmHg (DE 15,83). En personas con sólo HTA se encontró 125,9 mmHg (DE 15,0) y en aquellos con HTA + DM el promedio fue levemente mayor $(128,6 \mathrm{mmHg}$ DE 17,1$)$. Para la PAD el promedio global 80,34 mmHg (DE 11,4): en personas con sólo HTA fue $80,6 \mathrm{mmHg}$ (DE 10,6) y en usuarios con ambas condiciones fue 79,8 mmHg (DE 12,8). Por su parte, la HbA1c promedio fue $7.2 \%$ (DE 1,9) en DM y 7,4 \% (DE 1,9) en sujetos con ambas condiciones. (Tabla 1).

Tabla 2.

Distribución numérica y porcentual de fármacos utilizados por los participantes del estudio.

\begin{tabular}{|c|c|c|c|c|c|c|c|c|}
\hline & \multicolumn{2}{|c|}{$\begin{array}{c}\text { HTA } \\
(n=119)\end{array}$} & \multicolumn{2}{|c|}{$\begin{array}{c}\text { DM } \\
(n=34)\end{array}$} & \multicolumn{2}{|c|}{$\begin{array}{c}\text { HTA+DM } \\
(n=69)\end{array}$} & \multicolumn{2}{|c|}{ Total } \\
\hline & $n$ & $\%$ & $n$ & $\%$ & $\mathrm{n}$ & $\%$ & $n$ & $\%$ \\
\hline Ninguno fármaco & 32 & 26,9 & 13 & 38,2 & 12 & 17,4 & 57 & 25,7 \\
\hline 1 fármaco & 17 & 14,3 & 5 & 14,7 & 0 & 0 & 22 & 9,9 \\
\hline Entre 2 y 4 fármacos & 65 & 54,7 & 16 & 47,1 & 32 & 46,4 & 113 & 50,9 \\
\hline 5 y más fármacos & 5 & 4,2 & 0 & 0 & 25 & 36.2 & 30 & 13,5 \\
\hline \multicolumn{9}{|c|}{ Fármacos específicos prescritos } \\
\hline AINE & 19 & 16 & 6 & 17,6 & 33 & 47,8 & 58 & 26,1 \\
\hline \multicolumn{9}{|l|}{ Antagonista } \\
\hline Angiotensina I & 55 & 46,2 & 1 & 2,9 & 21 & 30,4 & 77 & 34,68 \\
\hline Antagonista del calcio & 12 & 10,1 & 0 & 0 & 10 & 14,5 & 22 & 9,91 \\
\hline Betabloqueador & 9 & 7,6 & 0 & 0 & 11 & 15,9 & 20 & 9 \\
\hline Biguanida & 17 & 14,3 & 20 & 58,8 & 46 & 66,7 & 83 & 37,39 \\
\hline Estatinas & 47 & 39,5 & 11 & 32,4 & 29 & 42 & 87 & 39,19 \\
\hline Fibratos & 4 & 3,4 & 1 & 2,9 & 4 & 5,8 & 9 & 4,05 \\
\hline Inhibidor de la ECA & 23 & 19,3 & 3 & 8,8 & 33 & 47,8 & 59 & 26,58 \\
\hline Insulina & 1 & 0,8 & 5 & 14,7 & 16 & 23,2 & 22 & 9,9 \\
\hline Nitrato & 0 & 0 & 0 & 0 & 1 & 1,4 & 1 & 0,45 \\
\hline Sulfonilureas & 6 & 5 & 5 & 14,7 & 19 & 27,5 & 30 & 13,5 \\
\hline Tiazidas & 35 & 29,4 & 0 & 0 & 25 & 36,2 & 60 & 27,03 \\
\hline
\end{tabular}


Utilización de fármacos: $25,7 \%$ de los usuarios no tenía registro de utilización de fármacos en los últimos 3 meses, más de la mitad $(50,9 \%)$ de las personas tenía registrado entre 2 y 4 fármacos. En primer lugar estuvieron estatinas, con $39,19 \%$, seguido de biguanidas con $37,39 \%$, antagonistas de la angiotensina I 34,68\%, tiazidas $27,03 \%$ e inhibidores de ECA 26,58\%. Sólo 9,9\% de los usuarios tiene indicado insulina de acción intermedia. (Tabla 2).

Objetivos terapéuticos: de las personas con HTA solo $61,7 \%$ tenía PAS y PAD controlada: de ellos, $62,2 \%$ correspondió a usuarios con sólo HTA y $60,9 \%$ a usuarios con HTA + DM. Respecto de los usuarios con DM, sólo $52,4 \%$ la tenían controlada. De ellos, 55,9\% correspondió a personas con sólo DM y 50,7\% a personas con HTA + DM. (Tabla 3).

\begin{tabular}{|c|c|c|c|c|}
\hline \multicolumn{5}{|c|}{$\begin{array}{l}\text { Tabla 3. Distribución porcentual, según logro del objetivo } \\
\text { terapéutico. }\end{array}$} \\
\hline Cobertura efectiva HTA & & $\begin{array}{l}\text { HTA } \\
(n=119)\end{array}$ & $\begin{array}{l}\text { HTA+DM } \\
(n=69)\end{array}$ & Total \\
\hline \multirow{2}{*}{$P A<140 / 90 m m h g$} & $\mathrm{n}$ & 74 & 42 & 116 \\
\hline & $\%$ & 62,2 & 60,9 & 61,7 \\
\hline \multirow[t]{2}{*}{$P A>140 / 90 m m h g$} & $\mathrm{n}$ & 45 & 27 & 72 \\
\hline & $\%$ & 37,8 & 39,1 & 38,3 \\
\hline \multicolumn{2}{|l|}{ Cobertura efectiva DMDM } & $\begin{array}{l}\text { HTA } \\
(n=34)\end{array}$ & $\begin{array}{l}\text { HTA+DM } \\
(\mathrm{n}=69)\end{array}$ & Total \\
\hline \multirow[t]{2}{*}{$\mathrm{HbA} 1 \mathrm{c}<\mathrm{a} 7$} & $n$ & 19 & 35 & 54 \\
\hline & $\%$ & 55,9 & 50,7 & 52,4 \\
\hline \multirow[t]{2}{*}{$\mathrm{HbA} 1 \mathrm{c}>$ igual a 7} & $\mathrm{n}$ & 15 & 34 & 49 \\
\hline & $\%$ & 44,1 & 49,3 & 47,6 \\
\hline
\end{tabular}

Evaluación del paciente sobre la atención de enfermedades crónicas: Globalmente los usuarios la evaluaron con puntaje promedio de 2,61 (DE 1,13). Lo mejor evaluado fue "Diseño de un sistema de entrega de cuidados/ Apoyo en la decisión" con un promedio de 3,24 (DE $1,25)$ y "Diseño de objetivos" con un puntaje promedio de 2,57 (DE 0,98). Lo peor evaluado fue la "Activación del paciente", con promedio 2,23 (DE 1,19). (Tabla 4).

Relación entre las variables del estudio con los indicadores de resultado clínico: en personas con HTA sólo se encontró relación significativa entre PAS y ${ }^{\circ}$ de fármacos $(r=0,204 ; p=0,026)$. Para la HbA1c de personas con DM se halló una relación significativa con años de
Tabla 4. Evaluación de la atención de enfermedades crónicas para pacientes.

\begin{tabular}{|l|r|r|}
\hline & Media & DE \\
\hline Activación del paciente & 2,23 & 1,19 \\
\hline $\begin{array}{l}\text { Diseño de un sistema de entrega de } \\
\text { cuidados/Apoyo en la decisión }\end{array}$ & 3,24 & 1,25 \\
\hline $\begin{array}{l}\text { Diseño de objetivos } \\
\text { Resolución de problemas considerando }\end{array}$ & 2,57 & 0,98 \\
\hline $\begin{array}{l}\text { contexto del paciente } \\
\text { Seguimiento-Coordinación }\end{array}$ & 2,56 & 1,32 \\
\hline Promedio global & 2,47 & 0,94 \\
\hline
\end{tabular}

Puntaje de 1 a 5: 1: nunca; 2: pocas veces; 3: a veces; 4 la mayoría de las veces; 5: siempre

enfermedad $(r=0,502 ; p=0,002)$. En usuarios con multimorbilidad (HTA+DM) se encontró relación significativa entre PAS con años de enfermedad $(r=0,309 ; p=0,01)$ y HbA1c con años de enfermedad $(r=0,309 ; p=0,01)$. (Tabla 5).

\section{Discusión:}

Este estudio muestra que en la selección aleatoria de usuarios hubo un marcado predominio de mujeres $(67,6 \%)$, concordante con que en Chile se controlan más mujeres que hombres ${ }^{6}$ con condiciones crónicas. El promedio de edad coincide con lo descrito por el Ministerio de Salud,

Tabla 5. Correlación (r) entre variables del estudio e indicadores de resultado clínico, según patología.

\begin{tabular}{lrrrrrrr} 
& \multicolumn{2}{c}{ HTA } & \multicolumn{1}{c}{ DM } & & \multicolumn{2}{c}{ HTA+DM } \\
& PAS & PAD & HbA1c & PAS & PAD & HbA1c \\
\hline Edad & 0,171 & $-0,062$ & 0,002 & 0,1 & $-0,01$ & 0 \\
Años enfermedad & 0,129 & 0,054 & $0,502^{* *}$ & $0,309^{*}$ & 0,2 & $0,309^{*}$ \\
$N^{\circ}$ fármacos & $0,204^{*}$ & $-0,008$ & 0,209 & 0,01 & $-0,06$ & 0,03 \\
Activación del paciente & $-0,141$ & 0,053 & $-0,038$ & $-0,01$ & 0,11 & $-0,16$ \\
Diseño de un sistema de & & & & & & \\
entrega de cuidados/Apoyo & & & & & & \\
en la decisión & $-0,142$ & $-0,033$ & 0,011 & $-0,06$ & 0,12 & $-0,12$ \\
Diseño de objetivos & $-0,098$ & 0,021 & $-0,219$ & $-0,07$ & 0,08 & $-0,1$ \\
Resolución de problemas & & & & & & \\
considerando contexto & & & & & & \\
del paciente & $-0,148$ & 0,044 & 0,021 & 0,06 & 0,05 & $-0,05$ \\
Seguimiento-Coordinación & $-0,001$ & 0,169 & 0,164 & $-0,08$ & $-0,08$ & $-0,15$ \\
Promedio global Evaluación & & & & & & & \\
de la atención de enfermedades & & & & & & & \\
crónicas & $-0,13$ & 0,066 & $-0,013$ & $-0,04$ & 0,06 & $-0,14$
\end{tabular}

$* p \leq 0,05, * * p \leq 0,01$ 
respecto de que, a mayor edad, mayor prevalencia de EC. En lo referente a la escolaridad, mayoritariamente se encontró enseñanza media en este grupo. Ello es relevante, ya que estudios dan cuenta de que una baja escolaridad puede estar asociada a malos hábitos de salud, mayor carga de enfermedad, baja adherencia al tratamiento y más complicaciones ${ }^{7,21-24}$.

Con relación al perfil de cronicidad, la HTA fue predominante, seguido de patología mixta (HTA + DM). Ello concuerda con que en Chile la HTA representa la principal carga de enfermedad, tanto en hombres como en mujeres $2,22,25$. Respecto de la patología mixta, la literatura muestra la existencia de patrones de multimorbilidad, como el hecho de que la HTA podría estar presente antes de que la persona desarrolle hiperglicemia, a través del síndrome metabólico. Así, otros hallazgos muestran que entre 50 y $80 \%$ de los usuarios con DM tenían, además, HTA y que usuarios con HTA tenían mayor probabilidad de desarrollar $\mathrm{DM}^{2,5}$ que los normotensos ${ }^{24-26}$.

En cuanto al número de medicamentos, más de la mitad de las personas $(50,9 \%)$ tenía prescrito entre $2 \mathrm{a} 4$ fárma$\cos$, lo que puede deberse a la multimorbilidad presente, que requiere de una serie de combinaciones farmacológicas y no farmacologías ${ }^{24}$ para el logro de los objetivos terapéuticos ${ }^{2}$. En relación a los fármacos prescritos, se encontró en primer lugar Estatinas, seguido de antagonistas de Angiotensina I, Biguanidas, Tiazidas e Inhibidores de la ECA. Este hallazgo concuerda con la farmacoterapia seleccionada para tratar HTA + DM que se ha demostrado que esta asociación reduce la tasa de ECV y la progresión de la enfermedad renal con muy pocos efectos adversos sobre el perfil lipídico y la homeostasis de la glucosa ${ }^{26}$. Por supuesto que para la efectividad de este tratamiento es necesario estimular al usuario para el cumplimiento terapéutico, así como también la adopción de hábitos de vida saludable ${ }^{9,27-29}$.

Otro punto importante corresponde a los indicadores de resultado clínico. En efecto, la PAS en DM es más elevada, posiblemente debido a una mayor rigidez arterial, lo que hace más difícil su control ${ }^{23}$. La evidencia revela asociación entre padecer DM y la generación de alteraciones microvasculares que pueden hacer incrementar valores de $\mathrm{PA}^{7,23}$. En lo que se refiere a la clasificación de los valores de PA, estos se encontraron en la categoría de PA normal. No obstante, se ha encontrado que el riesgo de ECV comienza a aumentar a niveles de PA 115/75 mmHg y se duplica con cada incremento de 20/10 mmHg ${ }^{25}, 30-33$. Los resultados de PAS, PAD y de HbA1c de este estudio fueron mejores que los encontrados por
Veliz ${ }^{21}$ y por Frei et al ${ }^{33}$. Respecto de la HbA1c los estudios dejan ver que la asociación de HTA con DM en el usuario puede llevar a mayor progresión de las complicaciones microvasculares ${ }^{32}$, retrasando la compensación de la enfermedad.

Respecto de la proporción de personas que alcanzaron los objetivos terapéuticos, se encontraron porcentajes menores a los alcanzados a nivel país $2015(65,8 \%)^{24}$. Esto se condice con lo encontrado por Garrido et al. ${ }^{7}$ quienes reportaron qué las personas con DM tienen menor \% de PA controlada, en contraste a la PA del no-diabético. En cuanto a las personas con DM, sólo la mitad de los usuarios tenia HbA1c $<7(52,4 \%)$. Cuando la DM coexistía con HTA los sujetos tenían menor cobertura $(50,7 \%)$ que aquellos con sólo DM $(55,9 \%)$. En este sentido, reducciones de $1 \%$ en la $\mathrm{HbA} 1 \mathrm{c}$ se ha asociado a una disminución de $21 \%$ en el riesgo de muerte asociado a la diabetes y $14 \%$ de reducción en el riesgo de infarto a 10 años plazo ${ }^{25,33}$. Es preciso incrementar la proporción de personas con EC bien controla en la región del Biobío, que en el año 2014 reportó una mortalidad por ACV superior al país $(59,3 \%)^{34}$. Para aquello será necesario valorar estrategias para cambiar la forma de otorgar cuidados, haciendo frente a las causas que podrían estar afectando el logro de la cobertura efectiva. Entre ellas destacan el escaso cumplimiento de estándares de manejo clínico, baja adherencia terapéutica, e inadecuadas competencias profesionales ${ }^{6,35}$.

Se consideró relevante, no sólo evaluar los parámetros clínicos del usuario externo, sino también evaluar la atención desde la perspectiva del usuario que recibe atención por el equipo de salud. En este sentido, se encontró que los usuarios evaluaron la atención con un promedio global de 2,61 (DE 1,13), que revela que "pocas veces" el equipo de salud realiza acciones para apoyar el automanejo de las personas con EC, dejando de manifiesto que el MCC requiere ser trabajado por los proveedores de atención de salud, para mejorar la práctica del cuidado y con ello la evaluación del cuidado crónico. La sub escala mejor evaluada fue "Diseño de un sistema de entrega de cuidados/Apoyo en la decisión". Este hallazgo evidencia que, aunque esta área no es comprendida en profundidad por el usuario, ellos valoran los esfuerzos realizados por el sistema sanitario en entregar una atención organizada. La segunda sub escala mejor evaluada correspondió a la "Diseño de Objetivos". La sub escala peor evaluada correspondió a "Activación del paciente", lo cual orienta al equipo a mejorar el empoderamiento y rol principal que debe tener el usuario y su familia en el logro de salud. Estos resultados son similares a los encontrados por Aragones et al. ${ }^{16}$ y Rick et al. ${ }^{17}$, 
aun cuando no se puede efectuar una comparación directa, debido a las diferencias en la estructura de los sistemas sanitarios. Pese a lo anterior, los datos reportados permiten advertir que las intervenciones tradicionales, tales como consultas y controles, educación y medicamentos, entre otros, no han logrado resolver el problema del cuidado de personas con EC.

En lo concerniente a la relación de las variables de estudio con la (s) EC de los participantes, para la HTA sólo se encontró relación entre PAS y $n^{\circ}$ de fármacos. Para la DM hubo relación significativa entre la HbA1c y los años de enfermedad y para a la multimorbilidad (HTA
+ DM) se encontró relación significativa entre HbA1c y años de enfermedad y PAS con años de enfermedad. No se encontró relación entre la evaluación de la atención y los indicadores de resultado clínico, según patología. Los resultados se condicen con los encontrados por Frei et $\mathrm{al}^{33}$. Estos hallazgos sugieren incorporar otras variables en futuras investigaciones, adicionales a las consideradas en este estudio. Entre ellas se incluirían la calidad de vida, parámetros antropométricos, y la adherencia terapéutica. Así se lograría determinar mejor los factores que podrían estar afectando el logro terapéutico en personas con $\mathrm{EC}^{35}$.

\section{Referencias}

1. ORGANIZACIÓN MUNDIAL DE SALUD. Cómo mejorar el manejo integrado de las enfermedades crónicas en el primer nivel de atención de los servicios de salud [Internet]. 2016 [citado 20 junio 2016]. Disponible en: http://iris.paho.org/xmlui/ handle/123456789/28486

2. MINISTERIO DE SALUD DE CHILE. Estrategia nacional de salud para el cumplimiento de los objetivos sanitarios para la década 2011-2020: elige vivir sano [Internet]. 2010 Abr [citado 25 abril 2015]. Disponible en: http://www.minsal.cl/portal/url/ item/c4034eddbc96ca6de0400101640159b8.pdf

3. CENTRO DE EPIDEMIOLOGÍA Y POLÍTICAS DE SALUD FACULTAD DE MEDICINA CLÍNICA ALEMANA- UNIVERSIDAD DEL DESARROLLO. Las enfermedades no transmisibles en Chile Aspectos Epidemiológicos y de Salud Pública [Internet]. 2016 [citado 15 mayo 2017]. Disponible en: http://medicina.udd.cl/centro-epidemiologia-politicas-salud/ files/2016/07/Serie-de-salud-poblacional-ECNT.pdf
4. MINISTERIO DE SALUD CHILE. CONSENSO 2014: Enfoque de riesgo para la prevención de enfermedades cardiovasculares [Internet]. 2014 [citado 20 junio 2016]. Disponible en: http://www.enfermeriaaps.com/portal/consenso-minsal-chile-2014-enfoque-de-riesgo-para-la-prevencion-de-enfermedades-cardiovasculare.

5. MINISTERIO DE SALUD DE CHILE. Guía clínica diabetes mellitus tipo 2. [Internet]. 2010 [citado17 marzo 2015]. Disponible en: http://www.minsal.cl.

6. PRADOS-TORRES A, CURA-GONZÁLEZ I, PRADOS-TORRES JD, LEIVA-FERNÁNDEZ F, LÓPEZ-RODRÍGUEZ JA, CALDERÓN-LARRAÑAGA A, et al. Multimorbilidad en medicina de familia y los principios Ariadne. Un enfoque centrado en la persona. Atención Primaria [Internet]. 2017 [citado 2 junio 2016]; 49(5):300-307. Disponible en: http://www.sciencedirect.com/science/article/pii/S0212656716304942 
7. GARRIDO J, CHACÓN J, SANDOVAL D, MUÑOZ R, LÓPEZ N, OYARZÚN E, et al. Control del Hipertenso, un desafío no resuelto: Avances logrados en Chile mediante el Programa de Salud Cardiovascular. Rev Chil Cardiol [Internet]. 2013 Julio 22 [citado 10 junio 2017]; 32(2): 85-96. Disponible en: http://www.scielo.cl/scielo.php?script=sci_ arttext\&pid=S0718-85602013000200001\&lng=es.

8. MINISTERIO DE SALUD DE CHILE. Modelo de atención para personas con enfermedades crónicas. Directrices para la Implementación. [Internet]. 2015 Jun [citado 27 mayo 2015]. Disponible en: http://www.enfermeriaaps.com/portal/modelo-de-atencion-para-personas-con-en.

9. STARFIELD B. POINT: The Changing Nature of Disease: Implications for Health Services. Medical Care [Internet] 2011 Nov [citado 10 mayo 2017]; 49 (11): 971- 972. Disponible en: http://www.jstor.org/stable/23053785.

10. RUBIERA LG, RIERA VR. Programa para mejorar la atención de las Enfermedades crónicas. Aplicación del Modelo De Cuidados para Enfermedades Crónicas. Aten Primaria. 2004; 34(4):206-9.

11. GLASGOW RE, WAGNER EH, SCHAEFER J, MAHONEY LD, REID RJ, GREENE SM. Assessment of Chronic Illness Care (PACIC) Med Care [Internet]. 2005 May [citado 20 abril 2017]; 43: 436-444.

12. WAGNER EH, AUSTIN B, DAVIS C, HINDMARSH M, SCHAEFER J, BONOMI A. Improving Chronic Illness Care: Translating Evidence into Action. Health Affairs [Internet]. 2001 Nov [citado 3 junio 2015]; 20 (6): 64-78.

13. BODENHEIMER T, WAGNER EH, GRUMBACH K. Improving Primary Care for Patients with Chronic Illness. JAMA [Internet]. 2002 Oct [citado 9 junio 2015]; 288 (14):17751779. Disponible en: https://www.ncbi.nlm.nih.gov/pubmed/12365965

14. SOLBERG LF, CRAIN L, SPERL-HILLEN J, HROSCIKOSKI M, ENGEBRETSON K, O'CONNOR P. Care Quality and Implementation of the Chronic Care Model: A Quantitative Study. Ann Fam Med [Internet]. 2006 Jul [citado 9 agosto 2015]; 4(4): 310-316.

15. GLASGOW RE, WHITESIDES H, NELSON CC, KING DK. Use of the Patient Assessment of Chronic Illness Care (PACIC) with diabetic patients: relationship to patient characteristics, receipt of care, and self-management. Diabetes Care [Internet]. 2005; 28: 2655-2661.

16. ARAGONES A, SCHAEFER E, STEVENS D, GOUREVITCH M, SHAH N, GLASGOW R. Validation of the Spanish
Translation of the Patient Assessment of Chronic Illness Care (PACIC) survey. Prev Chronic Dis [Internet]. 2008; 5: 113.

17. RICK J, ROWE K, HANN M, SIBBALD B, REEVES D, ROLAND M, et al. Psychometric properties of the patient assessment of chronic illness care measure: acceptability, reliability and validity in United Kingdom patients with long-term conditions. BCM Health Serv Res [Internet]. 2012; 12: 293.

18. WENSING M, J LIESHOUT J, JUNG H, HERMSEN J, ROSEMANN T. The Patients Assessment Chronic Illness Care (PACIC) questionnaire in The Netherlands: a validation study in rural general practice. Health Services Research [Internet]. 2008; 8: 182.

19. LAGOS ME, SALAZAR A, SALAS P. Adaptación y validación de la versión chilena del cuestionario "Evaluación de la Atención de Enfermedades Crónicas para pacientes”. Rev Med Chil. 2017; 145(7): 869-878.

20. RODRÍGUEZ EY. Comités de Evaluación Ética y Científica para la Investigación en Seres Humanos. 2004.

21. VELIZ L. Automedicación y adherencia terapéutica como determinantes del control integral de enfermedades cardiovasculares Tesis optar al grado de Doctor [Internet]. 2014 [citado 20 Junio 2016].

22. SOLIMANO G, MAZZEI M. ¿De qué mueren los chilenos hoy?: perspectivas para el largo plazo. Rev. méd. Chile [Internet]. 2007 Julio [citado 20 junio 2016]; 135(7): 932-938. Disponible en: http://www.scielo.cl/scielo.php?script=sci_arttext\&pid=S0034-98872007000700015\&lng=es.

23. ESCOBAR C, BARRIOS V, CALDERÓN A, LLISTERRI J, GARCÍA S, RODRIGUEZ G, et al. Diabetes in the hyperten-sive population under primary care in Spain. Blood pressure and lipids control. Rev Clin Esp [Internet].2007 Mayo [citado 20 junio 2016]; 207:221-227. Disponible en: http://www.revclinesp.es/es/diabetes-mellitus-poblacion-hipertensa-asistida/articulo/13102313/.

24. MINISTERIO DE SALUD CHILE. Fármacos básicos para el tratamiento farmacológico de la hipertensión arterial en Chile: Estandarización del tratamiento de la HTA en Chile [Internet]. 2014-2015 [citado 20 Junio 2016]. Disponible en: https:// es.scribd.com/document/337568408/Farmacos-Basicos-Para-El-Tratamiento-de-La-HTA-en-Chile-3.

25. ARAYA M. Hipertensión arterial y diabetes mellitus. Revista costarricenses ciencias médicas [internet]. 2004 [citado 20 agosto 2017]; 25: 3-4. Disponible en: http:// www.scielo.sa.cr/scieloOrg/php/articleXML.php?pid=S0253-29482004000200007\&amp;lang= 
26. PÉREZ I, RODRÍGUEZ F, DÍAZ E, CABRERA J. Mitos y realidad de la hemoglobina glucosilada. Med Int Mex [Internet]. 2009 [citado 20 Junio 2016]; 25(3):202-209. Disponible en: http://www.medigraphic.com/pdfs/medintmex/mim-2009/ mim093g.pdf

27. SMITH SM, WALLACE E, O’DOWD T, FORTIN M. Interventions for improving outcomes in patients with multimorbidity in primary care and community settings. Cochrane Database Syst Rev [Internet]. 2016 Mar [citado 20 abril 2017]; 14(3). Disponible en: https://www.ncbi.nlm.nih.gov/pub$\operatorname{med} / 2697652930$

28. WAGNER E. Counterpoint: chronic illness and primary care. Med Care [Internet]. 2011 Nov [citado 10 Mayo 2017]; 49 (11): 973- 975. Disponible en: http://www.jstor.org/stable/23053785

29. LORIG KR, SOBEL DS, RITTER PL, LAURENT D, HOBBS M. Effect of a self-management program on patients with chronic disease. Eff Clin Pract. 2001; 4(6):256-62.

30. ORGANIZACIÓN MUNDIAL DE LA SALUD. Séptimo Informe del Comité Nacional Conjunto de los Estados Unidos de América sobre Prevención, Detección, Evaluación y Tratamiento de la Hipertensión Arterial [Internet]. 2010 [citado 15 junio 2017].,
31. GRUNDY SM, BENJAMIN IJ, BURKE GL, CHAIT A, ECKEL RH, HOWARD BV, et al. Diabetes and Cardiovascular Disease A Statement for Healthcare Professionals from the American Heart Association. AHA Scientific Statement [Internet]. 1999 Sep 07 [citado 10 junio 2017]; 100 (10): 1132-1133.

32. STRATTON I, ADLER A, NEIL A, MATTHEWS D, MANLEY S, CULL C, et al. Association of glycaemia with macrovascular and microvascular complications of type 2 diabetes (UKPDS 35): prospective observational study. BMJ [Internet]. 2000; 321:405-412.

33. FREI A, HERZOG S, WOITZEK K, HELD U, SENN O, ROSEMANN T, et al. Characteristics of poorly controlled Type 2 diabetes patients in Swiss primary care. Cardiovasc Diabetol [Internet]. 2012; 11: 70.

34. MINISTERIO DE SALUD DE CHILE. Atlas de salud región del Bío-Bío [Internet]. 2013 [citado 20 mayo 2014].

35. ORGANIZACIÓN MUNDIAL DE LA SALUD. Preparación de los profesionales de la atención de salud para el siglo XXI: el reto de las enfermedades crónicas. Enfermedades No Transmisibles y Salud Mental Enfermedades Crónicas y Promoción de la Salud. Ginebra [Internet]. 2005 [citado 10 abril 2015]. 